\title{
Les ingrédients magiques de la relation d'aide
}

\section{Une exploration des facteurs à l'origine du succès de l'intervention dans le champ de la santé mentale}

\author{
Christiane BERGERON-LECLERC \\ Professeure \\ Département des Sciences Humaines \\ Université du Québec à Chicoutimi
}

Cécile CORMIER

Travailleuse sociale

Centre hospitalier Robert-Giffard - Institut universitaire

Cet article propose une exploration des facteurs contribuant au succès de l'intervention dans le champ de la santé mentale. Dans un premier temps, est proposé un survol de différentes stratégies utilisées par les travailleuses sociales afin de favoriser l'intégration sociale des personnes ayant des troubles mentaux graves. De façon plus spécifique, il est question de quatre courants théoriques ayant influencé la pratique du travail social des années 1960 jusqu'à nos jours: réinsertion sociale, réadaptation psychosociale ou psychiatrique, rétablissement et résilience. Dans un deuxième temps, sont présentés les résultats d'une démarche de consultation entreprise auprès des informateurs et informatrices clés du champ de la réadaptation en santé mentale, consultation qui a permis de dégager certains ingrédients sous-tendant les effets positifs de l'intervention, notamment la contribution de l'espoir à ce processus.

Mots-clés : Travail social, santé mentale, intégration sociale, espoir, relation d'aide, réadaptation psychiatrique, rétablissement, résilience.

This paper aims to explore the factors contributing to successful interventions in the field of mental health. First, we propose an overview of various strategies used by social workers in order to support the social integration of people with serious mental illnesses. In a more specific way, we review four theoretical approaches that having influenced the social work practice from the 60's until our days: social reinsertion, psychiatric rehabilitation, recovery and resiliency. In the second part, the results of a consultation with key informants involved in the field of psychiatric rehabilitation are presented. On that basis, we have identified some of the ingredients which underlie the positive effects of intervention, in particular the contribution of hope in this process.

Key Words: Social Work, mental health, social integration, hope, helping relationships, psychiatric rehabilitation, recovery, resiliency.

Service social - Volume 55, numéro 1, 2009 


\section{INTRODUCTION}

Existe-t-il des similitudes entre la pratique actuelle du travail social et celle qui prévalait dans les hôpitaux psychiatriques dans les années 1960? À travers le temps et les modes, les stratégies utilisées par les intervenantes afin de favoriser l'intégration des personnes ayant des troubles mentaux graves se sont-elles transformées? Et que sait-on des facteurs qui influencent favorablement l'intervention, appelés ici « ingrédients magiques », à ces processus? À travers cet article, nous proposons, dans un premier temps, une exploration de différentes avenues théoriques ayant marqué le champ de l'intervention en santé mentale. Ce survol des quatre « R », que sont la réinsertion sociale, la réadaptation psychosociale ou psychiatrique, le rétablissement et la résilience, permettra de faire ressortir les similitudes et les différences en ce qui concerne les façons actuelles et antérieures de promouvoir l'intégration sociale. Ayant un intérêt marqué pour les facteurs et les mécanismes à l'origine des effets de l'intervention, nous proposons, dans un deuxième temps, une incursion dans le «monde du magique ». Bien qu'elle puisse sembler inappropriée d'un point de vue scientifique, cette métaphore s'est révélée un outil fort prolifique lors d'un processus de consultation que nous avons entrepris auprès des informateurs et informatrices clés du champ de la réadaptation en santé mentale. La deuxième partie de l'article est donc consacrée aux résultats ayant émergé de ce processus exploratoire, afin de dégager certains des facteurs à l'origine du succès de la relation d'aide.

\section{Un survol des approches d'intervention : les quatre « $R$ »}

Depuis les années 1960, la pratique du travail social en santé mentale, au Québec et ailleurs, a comme visée l'adaptation réciproque de l'individu et de son environnement. Au fil des ans, bon nombre de travailleuses sociales ${ }^{1}$ ont contribué à l'amélioration du fonctionnement social et ultimement à l'intégration sociale des personnes ayant des troubles mentaux graves. Selon les époques et les courants théoriques en ce qui concerne l'intervention auprès des personnes ayant de tels troubles, différentes stratégies ont été privilégiées par les travailleuses sociales afin de créer des opportunités d’intégration à la vie en société. Et cela, dans des domaines aussi diversifiés que l'emploi, l'éducation, le logement, les loisirs, les relations sociales et les services sociaux et de santé. Bien que d'autres perspectives théoriques aient aussi influencé l'organisation des services et l'intervention dans le champ de la santé mentale au Québec, on traite ici des approches qui s’inscrivent dans la lignée directe du travail social, donc, de celles qui tiennent compte des interactions entre la personne et son environnement. Ainsi, il sera question des courants de la réinsertion sociale, de la réadaptation psychosociale ou psychiatrique, du rétablissement et de la résilience, que nous regroupons ici sous l'appellation « les quatre $\mathrm{R} »$.

1. Le féminin est utilisé dans le texte car une majorité de femmes exercent la profession de travail social. 


\section{La réinsertion sociale}

Le premier « $\mathrm{R}$ », pour réinsertion sociale, a pris naissance dans le processus de désinstitutionalisation amorcé au Québec dans les années soixante. Bien que l'émergence de ce processus résulte de l'interaction entre plusieurs facteurs, tels que les coûts élevés associés aux hospitalisations de longue durée et les espoirs engendrés par la découverte des neuroleptiques, le coup d'envoi a été donné lors de la parution du livre Les fous crient au secours (Pagé, 1963), qui décrie les conditions de vie asilaire de l'époque. Cette publication a eu un effet tel, qu'elle a été à l'origine de la mise sur pied de la commission Bédard-Lazure-Roberts (1962), de laquelle a découlé une série de recommandations quant à l'organisation des services, dont un moratoire sur la construction de nouveaux hôpitaux psychiatriques. Cette époque en est une où ont été mis en lumière les effets pervers de l'hospitalisation à long terme, notamment en ce qui a trait à l'autonomie des personnes, et où a été revalorisé le maintien dans la communauté, puisqu'il contribue à l'amélioration de la qualité de vie des personnes ayant des troubles mentaux graves. Ce dernier constat est appuyé par de nombreux écrits scientifiques parus à cette époque (McEwan et Goldner, 2001; Gouvernement du Québec, 1997c, dans Rodriguez et al., 2006). Il importe de noter cependant que cette augmentation de la qualité de vie serait davantage liée à la perception de liberté, qu'aux conditions objectives de vie des personnes à cette époque (Bachrach, 1970).

Afin de favoriser la réinsertion des personnes ayant des troubles mentaux, divers moyens visant le renforcement des capacités individuelles et environnementales ont été déployés. Dans ce contexte, des modules de développement des habiletés fonctionnelles et sociales ont été créés au sein des unités de soins psychiatriques. Cependant, le constat selon lequel il est difficile pour un individu de généraliser et d'appliquer dans la vie quotidienne des habiletés acquises dans un milieu artificiel (Stein et Test, 1980) a mené au développement d'alternatives, tels que les clubs psychosociaux dans la communauté. Sur le plan de la pratique du travail social, cette période a été caractérisée par un double mandat: celui de la protection et celui de l'intégration sociale (Bergeron-Leclerc et al., 2003). Certaines actions concrètes, telles que le développement des premières ressources de type familial (RTF), ont contribué à maximiser ce mandat de réinsertion.

\section{La réadaptation psychosociale ou psychiatrique}

Le deuxième « $\mathrm{R}$ », pour réadaptation psychosociale ou encore psychiatrique (selon que l'on réfère à la nature de l'intervention ou à l'incapacité de la personne), a émergé dans les années quatre-vingt. Le développement de cette perspective d'intervention résulte de la transposition - du champ de la santé physique vers celui de la santé mentale - du modèle de l'Organisation mondiale de la santé (OMS), en l'occurrence, la Classification internationale des déficiences, incapacités et handicaps [CIDIH] (OMS, 1980). Portée d'abord par l'équipe du Center for Psychiatric Rehabilitation de l'Université de Boston aux États-Unis, la notion de réadaptation, dans le champ de la santé mentale, a été introduite au Québec par l'Association 
québécoise pour la réadaptation psychosociale $(\mathrm{AQRP})^{2}$. Bien que plus facile à définir dans le champ de la réadaptation physique, la notion de réadaptation psychiatrique implique, elle aussi, une adaptation mutuelle de l'individu et de son environnement (Anthony et al., 2002). Sans cette adaptation réciproque, la personne ayant une incapacité psychiatrique, quelle qu'elle soit, se retrouve à risque d'exclusion sociale. Ainsi, afin de favoriser une pleine participation sociale chez les personnes ayant de telles incapacités, différentes stratégies d'intervention mettant l'accent sur le développement et l'utilisation des forces individuelles et des ressources disponibles dans l'environnement social sont nécessaires (Fougeyrollas et al., 1998). L'intervention vise donc l'augmentation du pouvoir individuel et de l'autonomie, en s'appuyant sur l'autodétermination de la personne. Pour ce faire, ont été créées différentes ressources de réadaptation dans la communauté, principalement dans les domaines du logement (par exemple, des appartements supervisés ou des foyers de groupe), du travail (par exemple, des organismes d'intégration au travail ou des centres de travail adaptés) et du loisir (par exemple, des centres de jour). Le principe voulant que la personne soit au centre de l'intervention qui la concerne, s'actualise notamment à travers des initiatives telles que l'élaboration d'un projet de vie et d'un plan d'intervention basés sur les choix de la personne qui utilise les services (Bernier et al., 2000); le tout, dans une perspective de développement et de participation optimale dans la communauté.

\section{Le rétablissement}

Le troisième « $\mathrm{R}$ » désigne l’approche du rétablissement. Ce mouvement a vu le jour dans les années quatre-vingt à la suite de la parution d'une part, d'écrits relatant les résultats d'études longitudinales où ont été observés des taux de rétablissement pouvant atteindre les $60 \%$ chez les personnes ayant des troubles mentaux graves (Allott et al., 2002), et, d'autre part, mettant en évidence des récits de personnes dont le parcours de vie est marqué par des améliorations significatives, notamment aux plans personnel et social (Lovejoy, 1984; Deegan, 1988; Leete, 1989). Le rétablissement ne signifie pas nécessairement la guérison au sens médical du terme; il correspond plutôt à «la capacité d'un individu de transcender les symptômes, les limites fonctionnelles et les handicaps sociaux rattachés à un trouble mental » (Provencher, 2002 : 39). Pouvant à la fois être considéré comme processus et résultat, le rétablissement implique la réappropriation du pouvoir de l'individu en tant que sujet et citoyen (Liberman et Kopelowicz, 2002). Autrement dit, ce processus implique un accroissement du pouvoir aux plans personnel, interpersonnel ou politique; le tout, en vue d'une participation sociale accrue chez la personne ayant un trouble mental. Même si plusieurs écrits indiquent que le fait de recevoir des services de réadaptation psychiatrique n'est pas un préalable au rétablissement (Anthony et al., 2002), il reste que certaines pratiques développées ces dernières années paraissent favoriser la réappropriation du pouvoir chez les individus (Sylverstein, 2000). Il s’agit, entre autres, (a) des mesures de soutien aux études, à l'emploi et au logement; (b) des ressources et des groupes d'entraide autogérés (par et pour); et (c) de l'intervention familiale. Fait intéressant, le Québec

2. $\quad$ Site internet : www.aqrp-sm.org 
s’est récemment doté d'un plan d'action en santé mentale faisant la promotion du rétablissement et de l'appropriation du pouvoir (MSSS, 2005). L'approche du rétablissement est donc largement appuyée dans les milieux de planification en santé mentale au Québec. Parmi les mesures proposées, on remarque une volonté très nette quant à l'embauche de travailleurs pairs-aidants au sein des équipes d'intervention, comme c'est le cas pour certaines équipes québécoises et une vaste majorité d’équipes ontariennes de suivi intensif dans la communauté (Musgrave, 2002).

\section{La résilience}

Le quatrième « $\mathrm{R}$ », pour résilience, est plus difficile à situer en matière de périodicité historique. Bien que ce concept ait été mis en lumière dès la fin de la Deuxième Guerre mondiale, à la suite de l'observation des personnes ayant survécu aux camps de concentration (Cyrulnik, 1999 et 2001) et des enfants ayant vécu des traumatismes importants (Cyrulnik, 2001), son utilisation est plus restreinte dans le champ du travail social en santé mentale. Cependant, parce que la notion de résilience implique une interface entre la personne et son environnement social (Cyrulnik, 1999), elle interpelle directement les travailleuses sociales. Par ailleurs, si l'on considère l'annonce du diagnostic ou encore l'hospitalisation comme des événements pouvant être marquants, voire traumatiques, la résilience touche directement les personnes ayant des troubles mentaux graves.

Pouvant être définie comme la capacité de rebondir et de s'épanouir malgré l'adversité (O’Leary, 1998), la résilience est à la fois un résultat et un processus qui peut s’apparenter au rétablissement. Nous avons cependant voulu en traiter distinctement puisqu'elle implique la notion d'événement traumatique de vie, de même que celles de facteurs de risque et de protection, qui sont très peu abordées dans les écrits sur le rétablissement. Ainsi, plus qu'une question de qualités individuelles ou génétiques, la résilience implique la présence de facteurs de protection individuels et environnementaux permettant le développement et l'épanouissement de la personne malgré la présence de risques (Masten et al., 1990). La résilience a pour fonction de protéger l'individu contre les répercussions dramatiques des événements traumatisants et, de façon plus spécifique: (a) de diminuer l'impact du risque; (b) de diminuer l'apparition de chaînes négatives; (c) d'établir et de maintenir l'identité et le sentiment d'efficacité personnelle; et (d) d'augmenter les opportunités (Rutter, 1987).

Concrètement, la résilience peut se manifester dans la société à l'aide de la création artistique ou de l'engagement social des personnes concernées. La créativité, étant perçue comme force réparatrice et source de valorisation, de reconnaissance et d'inclusion sociale, est encouragée depuis longtemps dans le domaine de l'intervention sociale. Cela est aussi vrai en ce qui concerne les personnes ayant des troubles mentaux, en raison de la croyance selon laquelle les activités artistiques constituent un formidable véhicule d'expression et d'épanouissement personnels (Andreoli, 1998, dans Lombardi 2005). Le programme d'accompagnement artistique 
Vincent et moi du centre hospitalier Robert-Giffard en est un bel exemple ${ }^{3}$. Outre la création artistique, cette capacité à rebondir face aux coups du sort peut amener la personne à choisir la voie du militantisme (Cyrulnik, 1999), à travers des groupes de défense des droits (par exemple, l'Association des personnes utilisatrices des services de santé mentale de la région de Québec $\left.[\mathrm{APUR}]^{4}\right)$. Dans certains cas, l'épanouissement des personnes sera tel qu'elles seront embauchées à titre de formatrices ou encore d'intervenantes au sein des équipes d'intervention.

\section{Points de repères méthodologiques : Et si l'espoir y était pour quelque chose?}

La synthèse des quatre « $\mathrm{R}$ » a permis de survoler quelques perspectives théoriques ayant eu une influence sur la pratique du travail social dans le champ de la santé mentale au Québec, au Canada et dans d'autres pays occidentaux (les États-Unis, principalement). Notre intérêt à explorer ces quatre courants d'idées réside dans la découverte des facteurs qui, au fil des ans, ont été identifiés comme étant à l'origine du succès de l'intervention, traduit ici en matière d'intégration sociale. Tout en étant conscientes du fait que l'obtention de résultats positifs dans le contexte de l'intervention résulte de l'interaction entre une multitude de facteurs, nous avons voulu explorer la contribution spécifique de l'espoir à ce processus. Ainsi, à la base de notre démarche exploratoire se retrouve la notion, appuyée par un nombre croissant d'écrits scientifiques, selon laquelle l'espoir émanant de la relation d'aide est une dimension essentielle au processus d'intégration sociale des personnes ayant des troubles mentaux graves. Et cela, qu'il s'agisse de l'espoir de pouvoir vivre dans la communauté (réinsertion sociale), de se développer malgré la présence d’une incapacité (réadaptation psychosociale ou psychiatrique), de participer individuellement ou collectivement à la vie en société (rétablissement) ou de s’épanouir dans divers rôles sociaux (résilience). Le tableau 1 résume les ancrages principaux des quatre approches eu égard à l'espoir.

3. Site internet : http://www.rgiffard.qc.ca/vincent_moi/index.asp

4. Site internet : http://www.apurquebec.org/ 
Tableau 1

L'espoir vu à travers les quatre « $\mathbf{R}$ »

\begin{tabular}{|l|l|l|l|}
\hline Concept & Mot-clé & Éléments de définition & $\begin{array}{l}\text { Espoir suscité par la } \\
\text { travailleuse sociale }\end{array}$ \\
\hline Réinsertion sociale & Maintien & $\begin{array}{l}\text { Retourner vivre dans la } \\
\text { communauté }\end{array}$ & $\begin{array}{l}\text { Espoir de vivre dans la } \\
\text { communauté }\end{array}$ \\
\hline $\begin{array}{l}\text { Réadaptation psycho- } \\
\text { sociale ou psychiatrique }\end{array}$ & Adaptation & $\begin{array}{l}\text { Développer les habiletés } \\
\text { personnelles et le soutien } \\
\text { environnemental nécessaires } \\
\text { à un meilleur exercice des } \\
\text { rôles sociaux }\end{array}$ & $\begin{array}{l}\text { Espoir de se développer } \\
\text { malgré la présence d'une } \\
\text { incapacité psychiatrique }\end{array}$ \\
\hline Rétablissement & Appropriation & $\begin{array}{l}\text { Transcender les symptômes, } \\
\text { les limites fonctionnelles et } \\
\text { les handicaps sociaux }\end{array}$ & $\begin{array}{l}\text { Espoir de devenir sujet et } \\
\text { citoyen }\end{array}$ \\
\hline Résilience & Épanouissement & $\begin{array}{l}\text { S'épanouir malgré } \\
\text { l'adversité }\end{array}$ & $\begin{array}{l}\text { Espoir de s'épanouir dans } \\
\text { divers rôles sociaux }\end{array}$ \\
\hline
\end{tabular}

L’introduction de la variable « espoir » prend racine dans plusieurs écrits concernant la motivation, notamment dans les travaux de Rogers (1975), qui stipulent que le changement émerge chez une personne lorsque celle-ci prend conscience de l'ampleur des difficultés engendrées par ses habitudes de vie (malaise) et qu'elle a une perception de pouvoir sur sa situation (espoir). Qui plus est, des écrits récents suggèrent que l'espoir, communiqué et suscité dans le contexte de la relation d'aide, est une source de motivation importante dans le processus de rétablissement des personnes ayant des incapacités psychiatriques (Russinova, 1999; Torrey et Wyzik, 2000). Russinova (1999) affirme même que la compétence des intervenantes à faire naître l'espoir optimise les résultats de l'intervention. Ainsi, elle distingue trois catégories de stratégies pouvant être utilisées par les intervenantes pour susciter cet espoir, et ultimement amener les personnes à se rétablir : (a) les stratégies visant à créer un espace relationnel propice à l'émergence de l'espoir (par exemple, croire au potentiel de la personne, l'accepter pour ce qu'elle est, l'écouter sans jugement, tolérer l'incertitude à propos de son avenir); (b) les stratégies amenant les personnes à reconnaître et mobiliser leurs forces personnelles (par exemple, aider la personne à se fixer et atteindre des objectifs, à développer de meilleures stratégies d'adaptation, à se remémorer des succès et des accomplissements antérieurs); et (c) les stratégies qui amènent les personnes à reconnaître et à utiliser les ressources environnementales, notamment, favoriser le soutien familial, favoriser les liens avec des personnes qui se sont rétablies, encourager la participation à des groupes d'entraide (Russinova, 1999). 


\section{Le démarrage et la réalisation d'un processus de consultation}

Afin d'alimenter notre réflexion eu égard au rôle de l'espoir, nous avons entrepris de consulter, à travers des groupes de discussion, des personnes évoluant dans le champ de la santé mentale, que ce soit à titre de personne utilisatrice de services, d'intervenante, de gestionnaire ou de proche. Au total, deux groupes de discussion ont été formés, l'un dans le cadre du XIIième Colloque de l'AQRP tenu à Rimouski à l'automne 2003 et l'autre, au cours de la même période, auprès des travailleuses sociales œuvrant au centre hospitalier Robert-Giffard (CHRG) ${ }^{5}$. Le groupe rencontré au colloque de l'AQRP était constitué de 41 personnes au total. Parmi ces personnes, $61 \%$ s'identifient comme des intervenantes, $14 \%$ comme des personnes utilisatrices de services, 9,8 \% comme des gestionnaires et 7,3\% comme des proches. Chez les intervenantes, $28 \%$ ont une formation en éducation spécialisée, $20 \%$ en sciences infirmières, $16 \%$ en travail social, $16 \%$ en intervention sociale (sans spécification), $8 \%$ en ergothérapie et $8 \%$ en psychiatrie. Par ailleurs, l'ensemble des intervenantes œuvre dans le champ de la réadaptation en santé mentale, qu'il s'agisse de suivi intensif dans la communauté, de soutien au logement, de soutien à l'emploi, ou autre. Le groupe de travailleuses sociales du CHRG était quant à lui d'une taille beaucoup plus petite, soit 7 personnes. Toutes les participantes ayant répondu à l'invitation travaillaient à ce moment-là dans des programmes intrahospitaliers. Il importe ici de souligner que la participation à chacun des groupes s'est effectuée sur une base volontaire. Pour cette raison, mais également parce que ce processus est de nature purement exploratoire, nous n'avons pas de prétention quant à la possibilité de généraliser les résultats obtenus ici.

Même s'il existe des variations quant à la composition des deux groupes de discussion et à la façon d'analyser les résultats obtenus, la méthode utilisée pour réaliser les deux entrevues de groupe a été similaire. Comme point de départ, un exercice de centration de quelques minutes a été réalisé dans le but d'aider les personnes participantes à se remémorer une expérience positive de relation aidant-aidé, une expérience de succès, de réussite. Partant de leur expérience concrète, les personnes participantes ont ensuite été invitées à répondre, par écrit dans un premier temps, puis à l'oral, à la question suivante : quels sont les ingrédients magiques, dans la relation aidant-aidé, qui ont été à l'origine de ce succès? Parce que l'usage de la métaphore est chose courante dans le contexte de l'intervention, et que les personnes consultées sont majoritairement des personnes intervenantes ou usagères, nous avons délibérément choisi d'utiliser le concept d'ingrédients magiques, parce que faisant appel à l'imaginaire, à l'expérience subjective des personnes, à des dimensions moins palpables de l'intervention. L'utilisation de cette métaphore est également liée à la consultation des écrits ayant démontré que les résultats de l’intervention dépendent à 40 \% de facteurs extrathérapeutiques (comprenant des éléments liés au hasard, mais

5. Nous tenons spécifiquement à remercier les membres du comité organisateur du XII ${ }^{\text {ième }}$ Colloque de l'AQRP ayant accepté la tenue d'un groupe de discussion le 23 octobre 2004; Marie-Luce Quintal, psychiatre, ayant marrainé l'invitation à participer au groupe de discussion de l'AQRP; Julie Lesage, directrice adjointe à la DSP (Centre hospitalier Robert-Giffard - Institut universitaire), alors Chef du Service social, ayant contribué à l'organisation des deux groupes de discussion; et l'ensemble des personnes participantes, pour leur temps et leur générosité. 
aussi des caractéristiques de la personne aidée), à $30 \%$ de la relation aidant-aidé, à $15 \%$ de l'orientation théorique de l'intervention et finalement à 15 \% de l'effet placebo (Miller, Duncan et Hubble, 2001). Enfin, l'usage de cette métaphore a généré des résultats forts intéressants, qui concernent, pour la plupart, la création d'un espace relationnel propice à l'émergence de l'espoir.

Notre plan initial concernant le déroulement des entrevues et l'analyse des données était de s’inspirer de la technique du groupe nominal (Delbecq et al., 1975). Cette technique, pensée pour des groupes idéalement constitués entre cinq et neuf personnes (le nombre maximal ne doit pas dépasser quinze), est une méthode d'entrevue structurée se déroulant selon la logique suivante : (a) d'abord, de façon individuelle, chaque personne répond par écrit à la question lancée, avec l'objectif d'identifier le plus d'idées possibles; (b) puis, en groupe, un remueméninges est effectué afin que chaque personne puisse émettre ses idées; (c) il s'ensuit une discussion concernant les idées émises, de façon à les clarifier, à les regrouper ou encore à les éliminer; et (d) cela mène à un vote individuel concernant les idées les plus importantes. À cette étape, les personnes doivent sélectionner les idées qu'elles jugent prioritaires et attribuer une cote numérique selon l'importance qu'elles y accordent ${ }^{6}$. Une fois le vote individuel réalisé, les données de chaque membre sont compilées et additionnées pour obtenir un résultat de groupe. Cette méthode a été retenue puisqu'elle permettait l'émergence de réponses multiples face à une question, l'expression libre de l'ensemble des personnes participantes et l'obtention d'un consensus de groupe face à la question posée; et le tout, à l'intérieur d'une seule rencontre. En ce qui concerne le groupe de travailleuses sociales du CHRG, cette méthode a été utilisée telle que décrite. Cependant, en raison du haut taux de participation pour le groupe de l'AQRP, il fut impossible d'utiliser cette méthode dans son intégralité. En effet, la période de discussion et de clarification des idées a été abrégée, puis celle du vote individuel transformée. À cette étape, il a été demandé à chacune des personnes de sélectionner et d'ordonner un maximum de dix ingrédients qui, selon elles, sont à l'origine du succès de l'intervention. Ce sont ensuite les chercheures responsables du projet qui ont attribué une cote d'importance à chacune des idées de façon à ce qu’émerge un résultat de groupe.

\section{Présentation et analyse des résultats}

\section{L'espoir comme ingrédient magique de la relation d'aide?}

L'espoir dont il est question ici est celui d'un mieux-être, celui d'un avenir favorable (Miller, 1992). Cet espoir peut être considéré comme un état de motivation positive impliquant une perception individuelle quant à sa capacité : (a) d’identifier des buts et de

6. Le nombre d'idées à sélectionner et à ordonner est fixé selon le nombre de personnes participant au groupe. Plus la taille du groupe est élevée, plus le nombre d'idées retenues est important, jusqu'à concurrence de neuf idées au maximum. Quant à la méthode de calcul, l'idée la plus importante se voit attribuer la cote numérique la plus élevée et vice-versa (s'il y a neuf idées, la cote de 9 sera attribuée à la plus importante et la cote de 1 à la moins importante). 
déterminer des moyens pour les atteindre; et (b) de se mettre en action afin d'actualiser le plan fixé précédemment (Snyder et al., 2002). Par ailleurs, même si plusieurs facteurs personnels, tels que le sentiment de compétence, la capacité d'adaptation et l'équilibre psychologique, sont nécessaires à la naissance de l'espoir, sans la présence d'une relation de partage et de confiance entre deux personnes, celui-ci ne peut émerger (Miller, 1992). Cette idée de mutualité revêt une importance cruciale et a été soulevée par plusieurs groupes d'auteurs (Lynch, 1974; Kirkpatrick et al., 1995; Russinova, 1999).

Au-delà de ces éléments de définition, certaines études réalisées dans le domaine de la psychothérapie révèlent l'importance de l'espoir et des attentes dans le processus d'intervention (Miller et al., 2001). Mais à notre grand étonnement, les résultats qui ressortent de notre processus de consultation ne vont pas dans ce sens. L'analyse des idées émergeant des groupes de discussion révèle plutôt que ce sont l'écoute, pour le groupe du CHRG, et la confiance, pour le groupe de l'AQRP, qui sont à l'origine des effets positifs de l'intervention. Le tableau 2 présente l'ensemble des résultats obtenus pour chacun des deux groupes. On constatera que ces résultats nous ramènent aux fondements et techniques de base de l'approche humaniste et nous rappellent l'utilité et la force thérapeutique d'une écoute de qualité (Rogers, 1951). Qui plus est, nos résultats rejoignent ceux d'autres études concernant l'importance de l'empathie, du respect, de la chaleur et de l'authenticité de l'intervenante dans le contexte de la relation d'aide (Patterson, 1984). Dans un même ordre d’idées, l'étude de Kirkpatrick et al. (1995) fait ressortir que des ingrédients tels que l'écoute, l'acceptation de la personne telle qu'elle est, la croyance dans le potentiel de l'individu et le fait de "prendre le temps", sont favorables au développement d'une relation qui sera porteuse d'espoir. Nos résultats, sans identifier directement l'espoir, nous mènent malgré tout à constater que la plupart des ingrédients énumérés par les participants constituent des préalables à l'établissement d'une relation permettant le développement de l'espoir. 
Tableau 2

Perception des personnes ayant participé aux entrevues de groupe à l'égard des ingrédients magiques de la relation d'aide ${ }^{7}$

\begin{tabular}{|ll|l|l|}
\hline Ingrédient & Fréquence & Somme \\
\hline Groupe de l'AQRP $(\mathrm{n}=41)$ & & \\
\hline 1 & Confiance & 20 & 45 \\
\hline 2 & Respect & 16 & 31 \\
\hline 3 & Écoute & 12 & 27 \\
\hline 4 & Empathie & 10 & 22 \\
\hline 5 & Relation égalitaire/réciprocité & 9 & 18 \\
\hline 6 & Croyance au potentiel individuel & 8 & 13 \\
\hline 7 & Importance d'être considéré comme une personne & 6 & 12 \\
\hline 8 & Authenticité & 5 & 8 \\
\hline
\end{tabular}

\begin{tabular}{|ll|l|l|}
\hline Groupe du CHRG $(\mathrm{n}=7)$ & & \\
\hline 1 & Écoute de ce que veut le client & 6 & 36 \\
\hline 2 & Respect mutuel & 6 & 33 \\
\hline 3 & Réappropriation du pouvoir & 6 & 29 \\
\hline 4 & Respect du rythme individuel & 4 & 26 \\
\hline 5 & Croyance au succès & 4 & 23 \\
\hline 6 & Authenticité & 4 & 22 \\
\hline 7 & Motivation client-intervenant & 5 & 21 \\
\hline 8 & Implication des réseaux de soutien & 6 & 15 \\
\hline
\end{tabular}

\section{Les valeurs du service social comme déterminants du succès de l'intervention?}

Au nombre des ingrédients identifiés par les participantes et participants des deux groupes, certains apparaissent directement liés aux valeurs fondamentales de la pratique du travail social. Il s'agit notamment : (a) de la croyance dans le potentiel de l'individu (croyance en la capacité humaine d'évoluer et de se développer, OPTSQ, 2000); (b) de l'implication des sous-systèmes (reconnaissance de la nécessité de percevoir et de comprendre l'être humain en tant qu'élément des systèmes interdépendants et potentiellement porteurs de changement, OPTSQ, 2000); (c) du respect du rythme individuel ou de l'importance de la relation égalitaire (respect de l'autonomie et de l'autodétermination de la personne, OPTSQ, 2000); et (d) de l'importance d'être considéré comme une personne (respect de la dignité de tout être humain, OPTSQ, 2000) $)^{8}$.

D'autre part, alors qu'à nos yeux il s'agit d'une condition évidente dans l'établissement de toute relation d'aide, nous avons été étonnées de constater que des participantes ont ressenti le

7. La colonne ayant pour titre Fréquence, témoigne du nombre de personnes ayant considéré l'énoncé comme important et celle intitulée Somme correspond à l'addition des résultats de chacun des répondants.

8. Cela rappelle l'importance d'insister sur ces aspects dans le cadre de la formation de baccalauréat en travail social, notamment à travers les stages réalisés par les étudiants. 
besoin de préciser l'importance d'être considéré comme une personne au nombre des ingrédients susceptibles de favoriser le succès de l'intervention. Faut-il en conclure que cet ingrédient est parfois absent de la prestation des services de santé et des services sociaux? Cela nous interpelle et mériterait que l’on s’y attarde davantage dans le cadre de recherches ultérieures.

\section{Le savoir-être : à l'origine du succès de l'intervention?}

La classification des ingrédients selon les trois types de savoir met en évidence l'importance du savoir-être dans la relation d'aide (voir le tableau 3). Ainsi, malgré la diversité des participantes et participants au plan de leur fonction ou de leur provenance, il y a consensus voulant que le succès de l'intervention soit lié à des valeurs, attitudes et comportements, que nous définissons comme étant le savoir-être. Loin de nous l'idée de nier l'importance des connaissances théoriques et des habiletés techniques des intervenants et intervenantes, cependant, ce sont les qualités d'être (ou l'utilisation consciente de soi), selon la perception des personnes répondantes, qui paraissent influencer la portée des interventions. Cela rejoint les écrits de Rodriguez et al. attestant que le savoir-être « est un incontournable, il détermine à lui seul la bonne utilisation des savoirs » (2006 : 268). Qui plus est, les résultats obtenus soutiennent l'idée que la qualité des pratiques en santé mentale est en partie associée à la primauté de la relation, en particulier la qualité de l'écoute et de l'accueil par l'intervenante (Rodriguez et al., 2006). Ces qualités, indispensables selon le point de vue des personnes utilisatrices de services en santé mentale, traversent aussi le savoir et le savoir-faire, mais sont difficilement évaluables par des mesures standards (Rodriguez et al., 2006). Nous sommes ici dans la sphère de l'intangible, d'où la nécessité de poursuivre des travaux de recherche alliant les méthodes quantitatives et qualitatives, afin d’obtenir une meilleure compréhension des processus associés aux effets de l'intervention.

Tableau 3

Classification des ingrédients magiques selon les trois types de « savoir "

\begin{tabular}{|c|c|c|}
\hline & Groupe de l'AQRP (n=41) & Groupe du CHRG $(\mathrm{n}=7)$ \\
\hline $\begin{array}{l}\text { SAVOIR } \\
\text { Connaissances théoriques }\end{array}$ & & - Réappropriation du pouvoir \\
\hline $\begin{array}{l}\text { SAVOIR-FAIRE } \\
\text { Capacités et aptitudes }\end{array}$ & & $\begin{array}{l}\text { - Motivation client-intervenant } \\
\text { - Implication des sous-systèmes }\end{array}$ \\
\hline $\begin{array}{l}\text { SAVOIR-ÊTRE } \\
\text { Attitudes et comportements }\end{array}$ & $\begin{array}{l}\text { - Confiance } \\
\text { - Respect } \\
\text { - Écoute } \\
\text { - Empathie } \\
\text { - Relation égalitaire/réciprocité } \\
\text { - Croyance au potentiel individuel } \\
\text { - Importance d'être considéré } \\
\text { comme une personne } \\
\text { - Authenticité }\end{array}$ & $\begin{array}{l}\text { - Écoute de ce que veut le client } \\
\text { - Respect mutuel } \\
\text { - Respect du rythme individuel } \\
\text { - Croyance au succès } \\
\text { - Authenticité }\end{array}$ \\
\hline
\end{tabular}




\section{CONCLUSION}

À travers cet article, nous avons exploré certains facteurs qui contribuent au succès de l'intervention dans le champ de la santé mentale. Cette exploration s'est faite en deux temps. D’abord, a été réalisé un bref examen de quatre perspectives théoriques ayant marqué la pratique du travail social dans ce domaine d'intervention. Ce survol des quatre « $\mathrm{R}$ » a permis de mettre en lumière la contribution de différentes stratégies d’intervention, notamment la capacité de susciter l'espoir dans le processus d'intégration sociale des personnes ayant des troubles mentaux graves. Puis, dans un deuxième temps, toujours dans le but d'alimenter cette réflexion à propos des ingrédients critiques de l'intervention, nommés ici les « ingrédients magiques », nous avons présenté les résultats d'une démarche de consultation entreprise auprès d'intervenantes œuvrant dans le champ de la santé mentale. En raison de la nature exploratoire de notre processus de consultation et des limites qui y sont associées, les résultats obtenus ne sont pas généralisables. Cependant, il en ressort clairement que la qualité des interactions dans le contexte d'une relation aidant-aidé est un facteur déterminant du succès de l’intervention. Il importe de souligner que les écrits existants vont dans le même sens : sans ce contexte relationnel favorable, l'espoir ne peut naître (Russinova, 1999); sans cet espoir, la motivation au changement ne peut émerger (Du Ranquet, 1983). Les résultats obtenus ici, s’ils ne sont pas totalement inédits, nous rappellent tout de même l'importance des facteurs fondamentaux de la relation d'aide, facteurs parfois oubliés sous la technique. Ils nous ramènent également à la nécessité d'un changement de paradigme en ce qui concerne l'intervention dans le champ de la santé mentale : passer d'une approche traditionnelle inspirée du modèle biomédical à une approche centrée sur les forces permettant aux personnes de regagner du pouvoir sur leur vie. Or tant les écrits que nos consultations auprès des acteurs du terrain nous montrent que ce n'est qu'à travers le développement d'une relation plus égalitaire entre la travailleuse sociale et la personne ayant un trouble mental que ce changement sera possible. Il y a donc lieu de promouvoir activement ce changement de philosophie dans nos pratiques ainsi que dans nos programmes de formation et dans les établissements offrant des services sociaux et de santé. 


\section{Bibliographie}

Allott, P., L. Loganathan, et K.W.M. Fulford (2004). « Discovering hope for recovery from a British perspective: a review of a selection of recovery literature, implications for practice and systems change », Canadian Journal of Community Mental Health, vol. 21, $n^{0}$ 3, p. 13-33.

Anthony, W.A., M. Cohen, M. Farkas, et C. Gagne (2002). Psychiatric Rehabilitation, ( $2^{\mathrm{e}}$ édition), Boston, MA: Center for Psychiatric Rehabilitation.

BACHRACH, L.E. (1970). Deinstitutionalization: An Analytic Review and Sociological Perspective, Rockville, MD: National Institute of Mental Health.

Bergeron-Leclerc, C., J. Lesage, S. Bellemare, V. Bergeron, D. Gosselin, et I. BOURDAGES (2003). « De la protection sociale au rétablissement : repères historiques et actuels concernant la pratique du service social au Centre hospitalier Robert-Giffard », Intervention, $\mathrm{n}^{0} 119$, p. 107-119.

Bernier, F., S. Dennie, J. Lesage, et L. Marchand (2000). Maître d'œuvre de mon projet de vie; guide interactif du plan d'intervention. Manuel à l'usage de l'intervenant et manuel à l'usage du participant, Québec, Centre Hospitalier Robert-Giffard.

CYRULNIK, B. (2001). Les vilains petits canards, Paris : Odile Jacob.

CYRULNIK, B. (1999). Un merveilleux malheur, Paris : Odile Jacob.

DEEgan, P. (1988). "Recovery: the lived experience of psychiatric rehabilitation », Psychosocial Rehabilitation Journal, vol. 6, nº 4, p. 11-19.

DelbecQ, A.L., A.H. VAn DE Ven, et D.H. Gustafson (1975). Group techniques for program planning: A guide to nominal group and delphi processes, Glenview (Illinois): Scott, Foresman.

Du RANQUET, M. (1983). Recherches en casework : l'efficacité et sa mesure en service social, St-Hyacinthe : Edisem.

Fougeyrollas, P., R. Cloutier, H. Bergeron, J. Côté, J., et G. St Michel (1998). Classification québécoise : processus de production du handicap, Lac St-Charles : RIPPH.

Kirkpatrick, H., J. Landeen, C. Byrne, H. Woodside, J. PAWlick, et A. Bernardo (1995). «Hope and schizophrenia: clinicians identify hope-instilling strategies», Journal of Psychosocial Nursing, vol. 33, n ${ }^{\circ}$ 6, p. 15-19.

LEETE, E. (1989). «How I perceive and manage my illness », Schizophrenia Bulletin, vol. 15, $n^{0} 2$, p. 197-200.

LiBERMAN, R.P., et A. KopelOWICZ (2002). « Recovery from schizophrenia: a challenge for the 21st century », International review of psychiatry, vol. 14, nº 4, p. 245-255. 
LOMBARDI, S. (2005). Champs libres. L'information psychiatrique, vol. 81, nº 4, p. 362-367.

LoveJOY, M. (1984). "Recovery from schizophrenia: a personal odyssey », Hospital and Community Psychiatry, vol. 35, nº 8, p. 809-812.

LYNCH, W.F. (1974). Images of hope: Imagination as the healer of hopeless, Notre-Dame: University of Notre-Dame Press.

MAsten, A.S., K.M. Best, et N. GARMEzy (1990). « Resilience and development: Contributions from the study of children who overcome adversity », Development and Psychopathology, vol. 2, p. 425-444.

Miller, J.F. (1992). "Inspiring hope », dans J.F. Miller Coping with chronic illness. Overcoming powerlessness, Philadelphia: FA Davis Company, p. 413-433.

Miller, S.D., B.L. Duncan, et M.A. Hubble (2001). Pour en finir avec Babel. À la recherche d'un langage unificateur pour l'exercice de la psychothérapie, St-Hyacinthe : Edisem.

Ministère DE LA SANTÉ ET Des Services sociaux (2005). Plan d'action en santé mentale: 2005-2010 « La force des liens », Québec.

MusgraVe, I. (2002). "Implementing assertive community treatment teams in Ontario. Of interest/applicability to Quebec? », Allocution prononcée dans le cadre du Colloque de l'AHQ ayant pour thème: Suivi intensif en équipe dans la communauté : Fidélité au modèle ACT et stratégie d'implantation, Montréal, 21 novembre 2002.

O'LEARY, V. (1998). « Strength in the face of adversity: individual and social thriving - thriving: broadening the paradigm beyond illness to health », Journal of Social Issues, vol. 54, n ${ }^{0} 2$, p. 425-445.

OPTSQ. (2000). Les valeurs fondamentales et les principes de la profession, [http://www.optsq.org/ordre/missionvaleur.html].

PATTERSON, C.H. (1984). « Empathy, warmth and genuineness in psychotherapy: a review of reviews », Psychotherapy, vol. 21, p. 431-438.

Provencher, H. (2002). «L'expérience du rétablissement : perspectives théoriques », Santé mentale au Québec, vol. 27, n 1, p. 35-64.

Rodriguez, L., L. Bourgeois, Y. LAndry, L. GuAy, et J.-L. Pinard (2006). Repenser la qualité des services en santé mentale dans la communauté. Changer de perspective, Sainte-Foy : PUQ.

Rogers, R.W. (1975). " A protection motivation theory of fears appeals and attitude change », Journal of Psychology, vol. 91, n 1, p. 93-114.

Russinova, Z. (1999). "Providers' hope-inspiring competence as a factor optimizing psychiatric rehabilitation outcomes », Journal of Psychiatric Rehabilitation, vol. 65, n ${ }^{\circ} 4$, p. 50-57. 
RUTTER, M. (1987). « Psychosocial resilience and protective mechanisms », American Journal of Orthopsychiatry, vol. 57, $\mathrm{n}^{0}$ 3, p. 316-331.

SNyder, C.R., K.L. RAND, et D.R. Sigmon (2002). «Hope theory. A member of the positive psychology family », dans C.R. SNYDER et S.J. LOPEZ, Handbook of positive psychology, New York: Oxford university press, p. 257-276.

StEIN, L.I., et M.A. TEST (1980). « An alternative to mental health treatment I: conceptual model, treatment program, and clinical evaluation », Archives of General Psychiatry, vol. 37, p. 392-397.

SylversteIN, S.M. (2000). "Psychiatric rehabilitation in schizophrenia: unresolved issues, current trends, and future directions », Applied and preventive psychology, vol. 9, p. 227248.

TORREY, W.C., et P. WYZIK (2000). « The recovery vision as a service improvement guide for community mental health center providers », Community Mental Health Journal, vol. 36, $\mathrm{n}^{\mathrm{o}}$ 2, p. 209-216. 